\title{
EVALUACIÓN DE LA ADIPOSIDAD CORPORAL SEGÚN ÍNDICE DE MASA CORPORAL Y CIRCUNFERENCIA DE CINTURA EN JÓVENES UNIVERSITARIOS
}

\author{
EVALUATION OF BODY ADIPOSITY ACCORDING TO BODY MASS \\ INDEX AND WAIST CIRCUMFERENCE IN YOUNG UNIVERSITY \\ STUDENTS
}

\author{
Catalina Vilchez-Avaca* \\ Cristián Alexis Silva Cancino ${ }^{* *}$ \\ Ana María Contreras Muñoz ${ }^{* * *}$ \\ María Matilde García Montecinos ${ }^{* * * *}$ \\ OSCAR RoJas JORQUERA ${ }^{* * * * *}$ \\ Rossana Gómez-CAmpos ${ }^{* * * * * *}$ \\ Marco Cossio-Bolaños ${ }^{* * * * * *}$
}

\begin{abstract}
RESUMEN
Objetivo: Analizar las diferencias de adiposidad corporal determinada por Indice de Masa Corporal (IMC) y la Circunferencia de Cintura (CC) según grupos de edad en jóvenes universitarios de ambos sexos. Material y método: Se evaluaron 330 alumnos universitarios, 152 varones y 178 mujeres de una Universidad de la zona central de Chile. Se conformaron tres grupos según edad (18 a 20 años, 21 a 23 años y 24 a 26 años) y se determinó la prevalencia de sobrepeso y obesidad según IMC y CC. Resultados: En las mujeres, tanto para IMC como para CC no hubo diferencias significativas entre los tres grupos de edad. En los hombres no hubo diferencias significativas en los tres grupos de edad por IMC, sin embargo sí hubo diferencias en la CC, pues el grupo de 18 a 20 años tuvo menor CC que los otros 2 grupos de edad $(\mathrm{p}<0,001)$. Según sexo y CC se observó mayor prevalencia de sobrepeso y obesidad en hombres $(\mathrm{p}<0,05)$. Con el IMC, no hubo diferencias entre ambos sexos $(\mathrm{p}>0,05)$. Conclusión: La CC parece ser un indicador más preciso que el IMC, ya que refleja aumentos significativos de obesidad abdominal a edades más avanzadas, al menos en varones universitarios, aunque es necesario desarrollar más estudios confirmatorios.
\end{abstract}

Palabras clave: Adiposidad, Índice de Masa Corporal, circunferencia de la cintura, jóvenes, enfermería.

\footnotetext{
* Profesora. Magíster (c) en Ciencias de la actividad física, Universidad Católica del Maule, Talca, Chile. Programa de Vida Saludable, Física Actividad y deportes. Universidad de Talca, UTAL, Talca, Chile. Email: catavilchez@hotmail.com

** Profesor. Magíster (c) en Ciencias de la actividad física, Universidad Católica del Maule, Talca, Chile. Programa de Vida Saludable, Física Actividad y deportes. Universidad de Talca, UTAL, Talca, Chile. Email: csilva.pef@hotmail.com

${ }^{* * *}$ Profesor. Magíster (c) en Ciencias de la actividad física, Universidad Católica del Maule, Talca, Chile. Programa de Vida Saludable, Física Actividad y deportes. Universidad de Talca, UTAL, Talca, Chile. Email: amariacontreras@gmail.com

${ }_{* * * *}$ Profesor. Magíster (c) en Ciencias de la actividad física, Universidad Católica del Maule, Talca, Chile. Programa de Vida Saludable, Física Actividad y deportes. Universidad de Talca, UTAL, Talca, Chile. Email: mgarciam@utalca.cl

${ }^{* * * * *}$ Profesor. Magíster (c) en Ciencias de la actividad física, Universidad Católica del Maule, Talca, Chile. Programa de Vida Saludable, Física Actividad y deportes. Universidad de Talca, UTAL, Talca, Chile. Email: oscarjorquerarojas@gmail.com

${ }_{* * * * * *}$ Doctora. Profesora Universidad Autónoma de Chile, Chile. Instituto de Actividad Física y Salud, Chile. Docente Universidad Nacional de San Agustín, Arequipa, Perú. Email: rossaunicamp@gmail.com

${ }_{* * * * * * *}$ Doctor. Profesor Departamento de Ciencias de la actividad física, Universidad Católica del Maule, Talca, Chile. Red de investigación en desarrollo biológico humano. Centro de investigación en desarrollo biológico humano, Talca, Chile. Email: mcossio1972@hotmail.com. Autor de correspondencia.
} 


\begin{abstract}
Objective: To analyze the differences in body adiposity determined by the Body Mass Index (BMI) and the Circumference of the Waist (WC) according to age groups in university students of both sexes. Material and method: 330 university students, 152 male and 178 female, from a University of the Central Zone of Chile, were evaluated. Three groups were formed according to age (18 to 20 years, 21 to 23 years and 24 to 26 years) and the prevalence of overweight and obesity was determined according to BMI and WC. Results: In women, both for BMI and WC there were no significant differences between the three age groups. In men there were no significant differences in the three age groups by BMI, however there were differences in WC, since the group of 18 to 20 years had lower WC than the other 2 age groups $(\mathrm{p}<0.001)$. According to sex and $\mathrm{WC}$, a higher prevalence of overweight and obesity was observed in men $(\mathrm{p}<0.05)$. Based on BMI, there were no differences between both sexes ( $\mathrm{p}>0.05$ ). Conclusion: WC appears to be a more accurate indicator than BMI, since it reflects significant increases in abdominal obesity at older ages, at least in male university students, although more confirmatory studies are needed.
\end{abstract}

Key words: Adiposity, Body Mass Index, waist circumference, youth, nursing.

Fecha recepción: 20/05/15 Fecha aceptación: 21/01/17

\section{INTRODUCCIÓN}

El tejido adiposo es una doble capa de piel que se dispersa por todo el cuerpo y los lugares más importantes de acumulación son la zona abdominal visceral, subcutánea abdominal y glútea femoral (1).

En la actualidad, la evaluación de la adiposidad corporal en niños, adolescentes y adultos es considerada relevante, puesto que, independientemente del método utilizado para medirla, contribuye a determinar el exceso de grasa corporal y a la identificación de los riesgos asociados a la salud en general. De hecho, los estudios epidemiológicos, a menudo, utilizan estas mediciones antropométricas para valorar la adiposidad corporal. Por lo general, se utiliza el Índice de Masa Corporal (IMC), pliegues cutáneos y perímetros de diversas regiones corporales (2), e incluso para complementar estos datos, también se evalúan algunas combinaciones de ellas, como la relación cintura-cadera, relación cintura-estatura, entre otras.

Básicamente en los adultos, las medicio- nes corporales se utilizan para evaluar la salud, la dieta, el riesgo de enfermedad, y los cambios en la composición corporal que se producen durante la vida adulta (3) que también pueden ser utilizados en forma cotidiana durante los controles de salud y puede servir como un medio de información científica para generar políticas públicas en el ámbito de la enfermería.

En ese contexto, los estilos de vida poco saludables de la población en general juegan un papel importante en los cambios de la composición corporal, no sólo a edades avanzadas, sino también en jóvenes, puesto que una reducción en la masa corporal magra y un aumento de la adiposidad corporal son producto del sedentarismo (4) y hábitos dietéticos inadecuados.

Actualmente varios estudios nacionales han reportado altas cifras de prevalencia de exceso de peso en jóvenes universitarios (58), por lo que se estima que la adiposidad corporal de esta población está experimentando cambios significativos en la forma del cuerpo.

Con todo, también se sabe que hay que 
considerar las diferencias de género en los depósitos de grasa en el cuerpo (9), pues las mujeres tienen mayor grasa abdominal subcutánea y menos grasa intrabdominal en relación con los hombres (10) y el IMC tendría dificultades para discriminar entre grasa corporal y masa magra (11), cuestión que debe tomarse en cuenta cuando se realicen clasificaciones de peso corporal (9).

Según lo anterior y partiendo de la hipótesis de que la CC podría ser una medición más precisa que el IMC, y que conforme la edad avanza podríamos tener algunos resultados diferenciados por edad y sexo, el objetivo del estudio fue analizar las diferencias de adiposidad corporal determinada por IMC y CC según grupos de edad en jóvenes universitarios de ambos sexos.

\section{MATERIAL Y MÉTODO}

Tipo de estudio y muestra: Se diseñó un estudio de tipo descriptivo, comparativo y transversal. La selección de la muestra fue no-probabilística (accidental) reclutada de forma voluntaria. Los criterios de inclusión fueron: rango de edad entre 18 a 26 años y estar debidamente matriculados en una universidad de la zona central de Chile, como alumnos regulares. Se excluyeron a los que no asistieron los días en que se programaron las evaluaciones y a los que presentaban problemas físicos-motores que impedían la evaluación antropométrica. El estudio tuvo la aprobación del Comité de ética de la Universidad Autónoma de Chile 048-2014. Todos los participantes firmaron el consentimiento informado y participaron 330 estudiantes, 152 varones y 178 mujeres.

Recolección de datos: Cuatro profesionales, con amplia experiencia, estuvieron encargados de obtener las medidas antropométricas de los participantes, en dependencias de la Universidad Autónoma de Chile adaptadas para la actividad, durante el mes de marzo de 2013. Para evaluar el peso, la estatura y la circunferencia de cintura se utilizó el protocolo estandarizado por Ross y Marfell-Jones (12). El peso corporal (kg) se evaluó con una balanza digital de marca Tanita con precisión de $100 \mathrm{~g}$ y una escala de 0 a $150 \mathrm{~kg}$. La Estatura $(\mathrm{cm})$ se evaluó a través de un estadiómetro de aluminio de marca Seca graduado en milímetros con una escala de 0 a 2,50 m. La CC $(\mathrm{cm})$ se midió en el punto medio entre la parte inferior de las costillas y la parte superior de la cresta iliaca mediante una cinta métrica no elástica de marca Seca milimetrada y con una precisión de $0,1 \mathrm{~cm}$. Para el IMC se utilizó la fórmula propuesta por Quetelet: [IMC=peso $(\mathrm{kg}) /$ estatura $\left.(\mathrm{m})^{2}\right]$ (13). Para clasificar por el IMC se adoptaron los puntos de corte de la National Heart, Lung, and Blood Institute (NHLBI) (14): bajo peso $<18,5 \mathrm{~kg} / \mathrm{m}^{2}$; normal entre $18,5-24,9$ $\mathrm{kg} / \mathrm{m}^{2}$; sobrepeso entre $25,0-29,9 \mathrm{~kg} / \mathrm{m}^{2} ; \mathrm{y}$ obesidad $\geq 30,0 \mathrm{~kg} / \mathrm{m}^{2}$. Respecto a los valores de la CC fue categorizada como sobrepeso en los varones cuando era $\geq 94 \mathrm{~cm}$ y en mujeres $\geq 80 \mathrm{~cm}$. Del mismo modo era obesidad en varones cuando los valores eran $\geq 102 \mathrm{~cm}$ y en mujeres $\geq 88 \mathrm{~cm}$ (15). Los jóvenes universitarios fueron distribuidos en tres grupos etarios: a) de 18 a 20 años, b) de 21 a 23 años y c) de 24 a 26 años.

Análisis estadístico: Se utilizó estadística descriptiva utilizando media aritmética, desviación estándar, frecuencias y porcentajes. Se verificó la normalidad de los datos mediante el test de Kolmoronov-Smirnov. Las diferencias entre proporciones se verificaron por $\mathrm{X}^{2}$. Para determinar las diferencias entre ambos sexos se utilizó $T$ de Student para muestras independientes. Las comparaciones entre los tres rangos de edad (grupos) fueron determinados por medio Anova (una vía) y la prueba de especificidad de Tukey. En todos los casos se adoptó un $\mathrm{p}<0,05$. Los datos se procesaron en SPSS 18,0 y Sigma Estat 8.0. 


\section{RESULTADOS}

Las características antropométricas y los estados nutricionales según IMC y CC se observan en la Tabla 1.

Los varones presentan mayor peso, estatura y CC en relación a las mujeres $(\mathrm{p}<0,001)$. No fueron significativas las diferencias en la edad e IMC $(p=0,0871)$ entre ambos sexos. En las prevalencias (frecuencias porcentuales) de estados nutricionales determinada por IMC, aunque hay diferencias porcentuales entre ambos sexos, estas no fueron significativas ( $\mathrm{p}=0,285)$. Respecto de las mediciones con CC, los varones presentaron mayor prevalencia de sobrepeso y obesidad respecto a las mujeres y estas diferencias sí resultaron significativas $(\mathrm{p}=0,029)$.

En las mujeres el IMC osciló entre 23,5 a $24,0 \mathrm{~kg} / \mathrm{m}^{2}$ y la CC entre 83,8 a $84,1 \mathrm{~cm} \mathrm{y}$, tanto para el IMC como para la CC, no se observó diferencias significativas entre los tres grupos de edad ( $p>0,05)$ (Figura 1). En los varones, en cuanto al IMC, no hubo diferencias entre los tres grupos de edad, los valores se mantuvieron constantes $(24,0$ a $25,5 \mathrm{~kg} /$ $\mathrm{m}^{2}$ ), sin embargo, en la CC sí se observaron diferencias significativas $(\mathrm{p}<0,05)$, donde los jóvenes del grupo de 18 a 20 años obtuvieron menor CC $(87,2 \pm 9,6 \mathrm{~cm})$ en relación a los otros dos grupos de edades superiores (21 a 23 años Media=91,1 DS 10,2cm y 24 a 26 años Media = 92,2 DS 9,6cm).

Tabla 1. Medidas antropométricas y estados nutricionales según IMC y CC en jóvenes universitarios, de una universidad de la zona central de Chile, $2013(\mathrm{~N}=330)$.

\begin{tabular}{|c|c|c|c|c|c|c|c|c|c|}
\hline \multirow[b]{2}{*}{ Variables } & & \multicolumn{2}{|c|}{$\begin{array}{l}\text { Varones } \\
(\mathrm{n}=152)\end{array}$} & \multicolumn{2}{|c|}{$\begin{array}{l}\text { Mujeres } \\
(\mathrm{n}=178)\end{array}$} & \multirow{2}{*}{$\begin{array}{c}\text { Tde } \\
\text { Student }\end{array}$} & \multirow{2}{*}{$p$} & \multicolumn{2}{|c|}{ Total } \\
\hline & & $\mathrm{X}$ & $\mathrm{DE}$ & $\mathrm{X}$ & $\mathrm{DE}$ & & & $\mathrm{X}$ & $\mathrm{DE}$ \\
\hline \multirow[t]{2}{*}{ Edad (años) } & & 20,9 & 2,2 & 20,7 & 2 & 0,8646 & 0,3879 & 20,8 & 2,1 \\
\hline & Peso (kg) & 74,3 & 12,6 & 62 & 10,5 & 9,672 & 0,001 & 68,2 & 11,6 \\
\hline \multirow{3}{*}{$\begin{array}{l}\text { Medidas } \\
\text { antropométricas }\end{array}$} & Estatura $(\mathrm{cm})$ & 173,6 & 6,4 & 161,1 & 5,7 & 18,763 & 0,001 & 167,4 & 6,1 \\
\hline & IMC $\left(\mathrm{kg} / \mathrm{m}^{2}\right)$ & 24,6 & 3,8 & 23,9 & 3,6 & 1,716 & 0,0871 & 24,3 & 3,7 \\
\hline & $\mathrm{CC}(\mathrm{cm})$ & 89,4 & 10 & 83,4 & 10,5 & 5,289 & 0,001 & 86,4 & 10,3 \\
\hline \multirow{6}{*}{$\begin{array}{l}\text { Estados } \\
\text { Nutricionales } \\
\text { según IMC }\end{array}$} & & fi & $\%$ & fi & $\%$ & $\chi^{2}$ & $p$ & fi & $\%$ \\
\hline & Bajo Peso & 2 & 1,3 & 6 & 3,4 & \multirow{4}{*}{3,79} & \multirow{4}{*}{0,285} & 8 & 2,4 \\
\hline & Normal & 91 & 59,8 & 117 & 65,7 & & & 208 & 63 \\
\hline & Sobrepeso & 45 & 29,7 & 39 & 21,9 & & & 84 & 25,5 \\
\hline & Obesidad & 14 & 9,2 & 16 & 9 & & & 30 & 9,1 \\
\hline & Total & 152 & 100 & 178 & 100 & & & 330 & 100 \\
\hline \multirow{5}{*}{$\begin{array}{l}\text { Estados } \\
\text { nutricionales } \\
\text { según CC }\end{array}$} & & fi & $\%$ & fi & $\%$ & $\chi^{2}$ & $p$ & fi & $\%$ \\
\hline & Normal & 91 & 59,8 & 131 & 72 & \multirow{3}{*}{7,02} & \multirow{3}{*}{0,029} & 222 & 67,3 \\
\hline & Sobrepeso & 45 & 29,7 & 35 & 21,3 & & & 80 & 24,2 \\
\hline & Obesidad & 16 & 10,5 & 12 & 6,7 & & & 28 & 8,5 \\
\hline & Total & 152 & 100 & 178 & 100 & & & 330 & 100 \\
\hline
\end{tabular}

$\mathrm{IMC}=$ Índice de Masa Corporal, $\mathrm{CC}=$ Circunferencia de la Cintura, DE= Desviación estándar. 
Figura 1. Valores de adiposidad corporal por grupos de edad en jóvenes universitarios según IMC y CC $(\mathrm{N}=330)$.
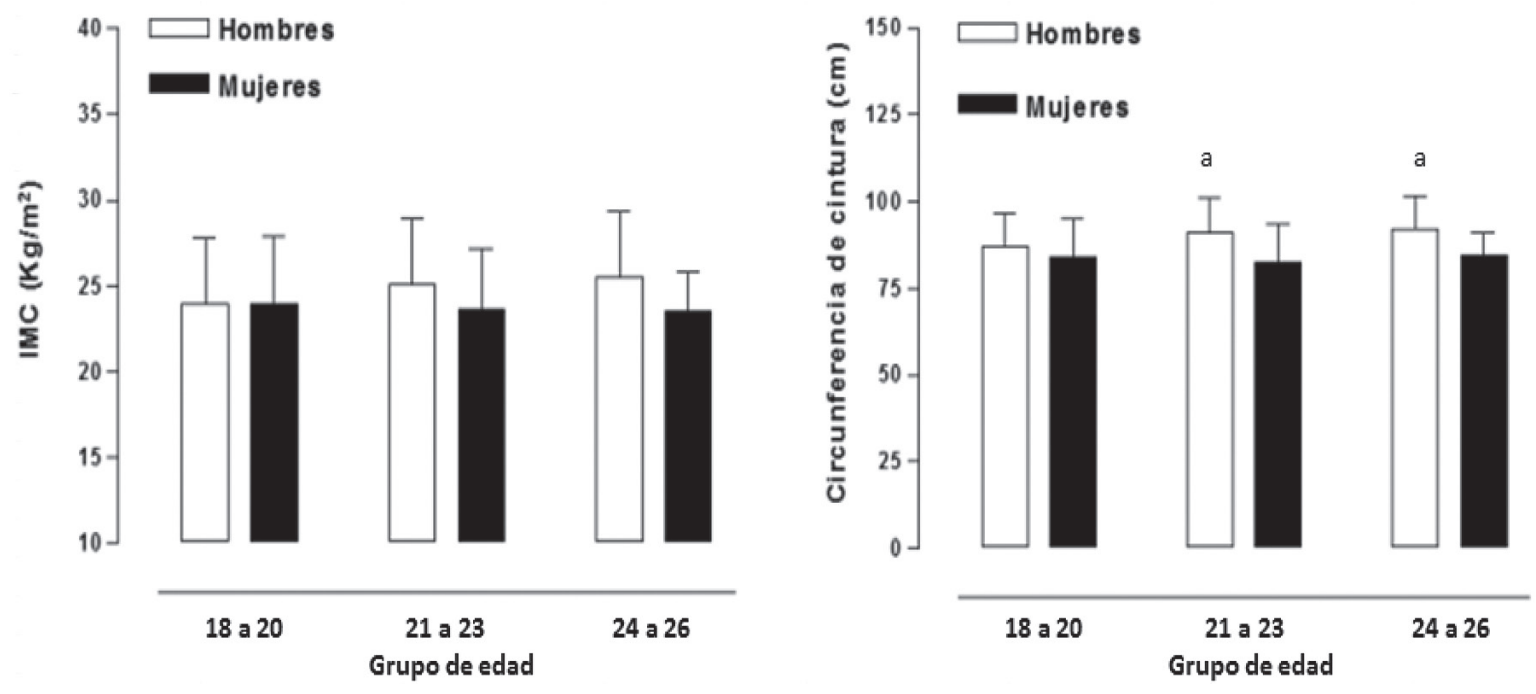

a: DS en relación al grupo de 18 a 20 años.

\section{DISCUSIÓN Y CONCLUSIÓN}

Los valores similares de IMC alcanzados en los tres grupos de edad y en ambos sexos concuerda con estudios anteriores, en el sentido de que este indicador no es una medida confiable para la adiposidad y sus limitaciones deben considerarse cuando se realicen clasificaciones de peso corporal $(9,10)$. En cuanto al incremento de la CC en los varones, respecto de los valores medios de adiposidad abdominal obtenidos en el segundo (21 a 23 años) y tercer grupo de edad (24 a 26 años), permite destacar que la CC sí resultó ser un indicador más real pues permitió precisar los grupos de edades en los que se presentaba la obesidad abdominal, que en este caso resultaron ser los jóvenes con edades más avanzadas.

El aumento de adiposidad abdominal observado en los varones de este estudio, probablemente se deba a condiciones genéticas e incluso medioambientales, pues el grado de exposición al medio ambiente influye en los patrones de las trayectorias de adiposidad abdominal (16).

En ese sentido, son varios los factores que podrían explicar los aumentos significativos de adiposidad abdominal en los varones, puesto que a menudo los jóvenes universitarios conviven con diversos fenómenos psicológicos, sociales, culturales, económicos (17) y situaciones propias del medio académico (18) que pueden afectar sus hábitos y que de algún modo podrían producir cambios importantes en la adiposidad abdominal. Llama la atención en este estudio, que son los varones quienes evidenciaron mayor adiposidad abdominal a edades más avanzadas, cuestión que concuerda con otros estudios recientes que sustentan que el exceso de peso que se observa en los jóvenes universitarios está asociado a los reducidos niveles de actividad física, sedentarismo y al mayor consumo de grasas en sus dietas $(19,20)$ que a futuro puede traer graves consecuencias para la salud. Aunque estudios anteriores han desarrollado estrategias pertinentes para mejorar las conductas alimenticias y estilos de vida saluda- 
bles en la población chilena en general (2123), al parecer estos esfuerzos no han sensibilizado a los jóvenes universitarios.

Por el contrario, cabe resaltar que el hecho de que las mujeres mantengan los valores promedios de la CC en los tres grupos de edad, probablemente se deba a aspectos estéticos (24), puesto que, en líneas generales, las mujeres están más preocupadas por su imagen corporal y son más conscientes de su peso corporal (25), incluso durante la juventud se someten a dietas de adelgazamiento, con el objetivo de mantener una imagen estilizada (26).

Respecto a la prevalencia comparada de sobrepeso y obesidad entre hombres y mujeres por medio de la CC, los hallazgos son consistentes con otros estudios, puesto que reportan que los varones presentan mayor prevalencia de sobrepeso y obesidad respecto a las jóvenes universitarias $(6,27,28)$. De hecho, estos resultados son una muestra clara que los jóvenes universitarios deben tomar conciencia de su imagen corporal, puesto que el excesivo aumento de la adiposidad abdominal podría llevar al deterioro de la salud nutricional, incluso en personas con un IMC normal (29). Por lo tanto, es necesario desarrollar estrategias de intervención en jóvenes basadas en el control alimentario y el ejercicio físico (30).

En cuanto a las limitaciones, no fue posible efectuar un muestreo probabilístico, esto impide la generalización de los resultados a otros contextos, así como también la falta de información respecto a los hábitos de alimentación no permitió analizar y comprender de mejor forma la adiposidad abdominal de los jóvenes estudiados; sin embargo, los resultados coinciden con otras investigaciones respecto de que la CC es un indicador altamente potencial para diagnosticar la adiposidad corporal, en especial de la región abdominal, lo cual debe ser considerado en futuros estudios y en diagnósticos de prevalencia de sobrepeso y obesidad en jóvenes universitarios.

A partir de los resultados obtenidos, este estudio también proporciona información relevante, en el que sugiere el uso de la CC en la evaluación realizada por enfermeras para valorar la adiposidad abdominal de los jóvenes y poder identificar casos específicos de sobrepeso y obesidad.

En conclusión, la CC parece ser un indicador más preciso que el IMC, ya que refleja aumentos significativos de obesidad abdominal a edades más avanzadas, al menos en varones universitarios, aunque son necesarios desarrollar más estudios de tipo transversal y longitudinal para confirmar estos hallazgos.

\section{REFERENCIAS}

1. Kopiczko A, Cieplińska J. General adiposity and adipose tissue distribution in young women from Warsaw. Journal of Medical Science. 2014; 2(83): 122-126.

2. Cossio-Bolaños MA, Pablos Abella C, Arruda M. Valoración de la adiposidad corporal de escolares en Arequipa, Perú. Rev Peru Med Exp Salud Pública. 2012; 29(4): 477-82.

3. Fryar CD, Gu Q, Ogden CL. Anthropometric reference data for children and adults: United States, 2007-2010. Vital Health Stat 11. 2012; (252): 1-48.

4. Schwartz RS. Obesity in the elderly. En: Bray GA, Bouchard C, James WPT, eds. Handbook of obesity. New York: Marcel Dekker, Inc.; 1998. p. 103-114.

5. Martínez MA, Leiva AM, Sotomayor C, Victoriano T, Von Chrismar AM, Pineda S. Factores de riesgo cardiovascular en estudiantes de la Universidad Austral de Chile. Rev Med Chil. 2012; 140(4): 426435.

6. Santos CS, Contreras AM, Bawarshi YA, Faúndez CC, Fuentes E.Q, Palomo IG. Anthropometric characteristics, physical fitness and lifestyles of university students in the Maule Region of Chile. Gazz Med Ital. 2013; 172 (6): 487-94. 
7. Vera-Villarroel P, Piqueras JA, Kuhne W, Cuijpers P, Van Straten A. Differences between men and women in self reported body mass index and its relation to drug use. Subst Abuse Treat Prev Policy [Internet]. 2014 [citado 2 enero 2014]; 9:1. Disponible en: https://substanceabusepolicy.biomedcentral.com/articles/10.1186/1747-597X-9-1.

8. Cossio-Bolaños MA, Vilchez-Avaca C, Contreras-Mellado V, Andruske C, Gómez-Campos R. Changes in abdominal obesity in Chilean university students stratified by body mass index. BMC Public Health [Internet]. 2016 [citado 9 dic 2015]; 16(33): 1-6. Disponible en: https://www.ncbi.nlm.nih.gov/pmc/articles/PMC4712471/pdf/12889_2015_Article_2587.pdf

9. Del Campo Cervantes JM, González González L, Gámez Rosales A. Relación entre el índice de masa corporal, el porcentaje de grasa y la circunferencia de cintura en universitarios. Investigación y Ciencia. 2015; 23(65): 26-32.

10. Peltz G, Aguirre MT, Sanderson M, Fadden MK. The role of fat mass index in determining obesity. Am J Hum Biol. 2010; 22(5): 639-647.

11. Zeng Q, Dong SY, Sun XN, Xie J, Cui Y. Percent body fat is a better predictor of cardiovascular risk factors that body mass index. Braz J Med Biol Res. 2012; 45(7): 591-600.

12. Ross WD, Marfell-Jones MJ. Kinanthropometry. En: MacDougall JD, Wenger HA, Geen HJ (eds). Physiological testing of elite the high-performance athlete. 2nd ed. Champaign, Illinois: Human Kinetics; 1991. p. 233-306.

13. Garrow JS, Webster J. Quetelet index (W/ $\mathrm{H}^{2}$ ) as a measure of fatness. Int J Obes. 1985; 9(2): 147-53.

14. National Heart, Lung, and Blood Institute (NHLBI). Clinical guidelines on the identification, evaluation, and treatment of overweight and obesity in adults: executive summary. Expert Panel on the Identification, Evaluation, and Treatment of Overweight in Adults. Am J Clin Nutr. 1998; 68(4): 899-917.

15. National Cholesterol Education Program (NCEP) Expert Panel on Detection, Evaluation and treatment of High Blood Cholesterol in Adults (Adult Treatment Panel III). Third Report of the National Cholesterol Education Program (NCEP) Expert Panel on Detection, Evaluation and Treatment of High Blood Cholesterol in Adults (Adult Treatment Panel III) final report. Circulation. 2002; 106(25): 3143-3421.

16. Shaw RJ, Green MJ, Popham F, Benzeval M. Differences in adiposity trajectories by birth cohort and childhood social class: evidence from cohorts born in the 1930s, 1950s and 1970s in the west of Scotland. J Epidemiol Community Health. 2014; 68(6): 550-556.

17. Vargas-Zárate M, Becerra-Bulla F, Prieto-Suárez E. Evaluación Antropométrica de estudiantes universitarios en Bogotá, Colombia. Rev Salud Publica. 2008; 10(3): 433-442.

18. Cota RP, Miranda LS. Associação entre constipação intestinal e estilo de vida em estudantes universitários. Rev Bras Nutr Clin. 2006; 21(4): 296-301.

19. Church TS, Thomas DM, Tudor-Locke C, Katzmarzyk P, Earnest C, Rodarte R, et al. Trends over 5 decades in U.S. occupation-related physical activity and their associations with obesity. PLoS One [Internet]. 2011 [citado 25 may 2011]; 6(5): e19657. Disponible en: https://www.ncbi. nlm.nih.gov/pmc/articles/PMC3102055/

20. Espinoza L, Rodríguez F, Gálvez J, McMillan N. Hábitos alimentarios y de actividad física en universitarios. Rev Chil Nutr. 2011; 38(4): 458-65.

21. Zacarías I, Pizarro T, Rodríguez L, González D, Domper A. Programa «5 al día» para promover el consumo de verduras y frutas en Chile. Rev Chil Nutr. 2006; 33 
(Suppl. 1): 276-80.

22. Castillo A, Smith C, Hirsch S, Brito A. ¿Es efectiva la consejería para aumentar el consumo de frutas y verduras y disminuir el riesgo cardiovascular en prevención secundaria?: una revisión. Rev Chil Nutr 2008; 35(2): 123-9.

23. Kain J, Leyton B, Cerda R, Vio F, Uauy R. Two-year controlled effectiveness trial of a school-based intervention to prevent obesity in Chilean children. Public Health Nutr. 2009; 12(9): 1451-61.

24. Trejo PM, Castro D, Facio A, Mollinedo FE, Valdez G. Insatisfacción con la imagen corporal asociada al Indice de Masa Corporal en adolescentes. Rev Cubana Enfermer 2010; 26(3): 150-60.

25. Madrigal-Fritsch H, De-Irala-Estévez J, Martínez-González MA, Kearney D, Gibney M, Martínez-Hernández JA. Percepción de la imagen corporal como aproximación cualitativa al estado de nutrición. Salud Publica Mex.1999; 41(6): 479-86.

26. Arroyo Izaga M, Rocandio-Pablo A; Ansotegui-Alday L. Imagen corporal en estudiantes universitarios: comportamien- tos dietéticos y actitudes nutricionales. BIBLID. 2007; 8: 199-215.

27. Mc Coll P, Amador M, Aros J, Lastra A, Pizarro C. Prevalencia de factores de riesgo de enfermedades crónicas no transmisibles en estudiantes de medicina de la Universidad de Valparaíso. Rev Chil Pediatr. 2002; 73(5): 478-82.

28. Bunker SJ, Colquhoun DM, Esler MD, Hickie IB, Hunt D, Jelinek VM, et al. "Stress" and coronary heart disease: psychosocial risk factors. Med J Aust. 2003; 178(6): 272-6.

29. Lee S, Bacha F, Gungor N, Arslanian SA. Waist circumference is an independent predictor of insulin resistance in black and white youths. J Pediatr. 2006; 148(2): 188-219.

30. Zea-Robles AC, León-Ariza H, Botero-Rosas DA, Afanador-Castañeda HD, Pinzón-Bravo LA. Factores de riesgo cardiovascular y su relación con la composición corporal en estudiantes universitarios. Rev Salud Publica (Bogotá). 2014; 16(4): 505-515. 